\title{
Administration of end-of-life drugs by family caregivers during covid-19 pandemic
}

\author{
Doing this safely needs training, support, and careful prescribing
}

\author{
Ben Bowers PhD student ${ }^{1}$, Kristian Pollock professor ${ }^{2}$, Stephen Barclay general practitioner ${ }^{1}$
}

${ }^{1}$ Primary Care Unit, Department of Public Health and Primary Care, University of Cambridge, Cambridge, UK; ${ }^{2}$ Nottingham Centre for the Advancement of Research into Supportive, Palliative and End of Life Care, School of Health Sciences, University of Nottingham, Nottingham, UK

Doctors, nurses, and family caregivers worldwide are facing tough decisions concerning the supply and administration of medications to manage symptoms when patients are dying from covid-19 or other conditions in the community or care homes. Proposed changes in practice aimed at ensuring adequate end-of-life symptom control need careful consideration alongside appropriate training and support.

Updated UK advice, including NICE rapid guidance on managing covid-19 symptoms in the community, reiterates the importance of prescribing medications in advance of need for pain, nausea and vomiting, agitation, and respiratory secretions. ${ }^{1-4}$ These drugs may be administered if needed by visiting doctors or nurses, as is already well established in some countries. ${ }^{4-6}$ However, this practice is being overhauled radically in response to the pandemic. ${ }^{1-3}$

Prescribers are now being asked to consider drug administration by family caregivers when community nurses and doctors are not available to administer end of life drugs in a timely way. ${ }^{1-3}$ Family caregivers willing to take on this role should be adequately trained and responsively supported with access to 24 hour phone advice. ${ }^{37-9}$

\section{Risks for family caregivers}

Prescriptions may need to include drug formulations that family caregivers can administer buccally, sublingually, or rectally, in addition to the subcutaneous injection route, as these are easier for non-professionals. ${ }^{12}$ There is good evidence for the effectiveness of subcutaneous injections of drugs such as opioids and midazolam at the end of life. ${ }^{41011}$ The buccal and sublingual routes are much less commonly used, however, and their limited evidence base comes primarily from professional experience and paediatric palliative care. ${ }^{11}{ }^{12}$ Both rectal and buccal routes in covid-19 carry the risk of transmitting infection. ${ }^{13}$

Although family caregivers commonly administer anticipatory medications in rural Australia, it is rare in the UK and many other countries. ${ }^{135}$ This is a big ask. Family caregivers may feel under pressure to undertake tasks for which they do not feel prepared or confident. ${ }^{14}$ They may feel a tension between their emotional involvement and this clinical task. Clinicians often worry that they may have hastened death if a patient dies shortly after drug administration. ${ }^{615}$ This anxiety may be even greater for family caregivers, with some worrying that it amounts to euthanasia. These tensions require sensitive explanation from the outset ${ }^{51416}$ since if the relative dies during the pandemic families will be confronting these concerns while grieving in isolation.

\section{Timely supply}

Increasing demand for drugs to control symptoms is also affecting prescribing decisions. ${ }^{1}$ The dilemma is whether to continue to prescribe anticipatory medications ahead of expected death, risking exhausting pharmacy supplies, or to delay until a patient is clearly dying. Acting to protect limited drug stocks for those who need them most risks delays in care and gives little time to prepare family caregivers to administer drugs. NICE guidance recommends that drugs continue to be prescribed ahead of need but in small quantities and cautions that patients with covid-19 can deteriorate rapidly so it is better to be prepared. ${ }^{1}$ This advice to continue judicious prescribing of anticipatory medications seems wise, provided that drug stocks remain adequate.

It would be helpful if community healthcare services could hold central stocks of the common end-of-life drugs, enabling rapid prescribing and dispensing. In care homes, allowing drugs prescribed for one resident to be used for another resident would ensure efficient use of limited supplies but would need legislative changes in many countries.

The pandemic is creating considerable challenges for end-of-life care across the world. It is vital to plan and provide suitable support now if changes in practice in the community are going to be safe and appropriate for patients and their family caregivers.

Competing interests: The BMJ has judged that there are no disqualifying financial ties to commercial companies. The authors declare the following other interests: 
$\mathrm{BB}, \mathrm{KP}$ and $\mathrm{SB}$ are researching end-of-life anticipatory prescribing practice. $\mathrm{BB}$ is funded by the National Institute for Health Research (NIHR) School for Primary Care Research. SB is supported by the NIHR Applied Research Collaboration East of England (ARC EoE) programme. The views expressed are those of the authors and not necessarily those of the NHS, the NIHR, or the Department of Health and Social Care.

Provenance and peer review: Not commissioned; externally peer reviewed.

1 National Institute for Health and Care Excellence. COVID-19 rapid guideline: managing symptoms (including at the end of life) in the community [NG163]. $2020 \mathrm{https} / / \mathrm{www}$.nice. org.uk/guidance/ng163/resources/covid19-rapid-guideline-managing-symptoms-includingat-the-end-of-life-in-the-community-pdf-66141899069893

2 Health Improvement Scotland. Alternatives to regular medication normally given via a syringe driver when this is not available, 9 Apr 2020. https://www.palliativecareguidelines. scot.nhs.uk/guidelines/symptom-control/alternatives-to-regular-medication-normally-givenvia-a-syringe-pump-when-this-is-not-available.aspx

3 Poolman M, Shuler A. GIG Cymru NHS Wales. CARer-Administration of as needed subcutaneous medication for common breakthrough symptoms in home-based dying people in wales (the CARiAD package). 2020. https://www.wales.pallcare.info/files/docs/ CARiAD\%20Package/A\%20-\%20CARiAD\%20for\%20Covid-19\%20policy\%20v1.0\%2020\% 20March\%202020.pdf

4 National Institute for Health and Care Excellence. Care of dying adults in the last days of life [NG31]. 2015. https://www.nice.org.uk/guidance/ng31/resources/care-of-dying-adultsin-the-last-days-of-life-pdf-1837387324357

5 Bowers B, Ryan R, Kuhn I, Barclay S. Anticipatory prescribing of injectable medications for adults at the end of life in the community: a systematic literature review and narrative synthesis. Palliat Med 2019;33:160-77. 10.1177/0269216318815796 30513254

6 Wilson E, Morbey H, Brown J, Payne S, Seale C, Seymour J. Administering anticipatory medications in end-of-life care: a qualitative study of nursing practice in the community and in nursing homes. Palliat Med 2015:29:60-70.10.1177/0269216314543042 25070861

7 Healy S, Israel F, Charles MA, Reymond L. An educational package that supports laycarers to safely manage breakthrough subcutaneous injections for home-based palliative care patients: development and evaluation of a service quality improvement. Palliat Med 2013:27:562-70. 10.1177/0269216312464262 23175512

8 Poolman M, Roberts J, Byrne A, etal . CARer-ADministration of as-needed subcutaneous medication for breakthrough symptoms in homebased dying patients (CARiAD): study protocol for a UK-based open randomised pilot trial. Trials 2019;20:105. 10.1186/s13063-019-3179-9 30732624

9 Cambridgeshire and Peterborough Clinical Commissioning Group. Palliative care prescribing. https://www.cambridgeshireandpeterboroughccg.nhs.uk/health-professionals/ prescribing-information/controlled-drugs-and-palliative-care/palliative-care-prescribing/

10 Jansen K, Haugen DF, Pont L, Ruths S. Safety and effectiveness of palliative drug treatment in the last days of life - a systematic literature review. J Pain Symptom Manage 2018;55:508-521.e3. 10.1016/j.jpainsymman.2017.06.010 28803078

11 Twycross R, Wilcock A, Howard P. Palliative care formulary. 6th ed. Pharmaceutical Press, 2018:153, 671.

12 Yap R, Akhileswaran R, Heng CP, Tan A, Hui D. Comfort care kit: use of nonoral and nonparenteral rescue medications at home for terminally ill patients with swallowing difficulty. J Palliat Med 2014;17:575-8. 10.1089/jpm.2013.0364 24708221

13 Xiao F, Tang M, Zheng X, Liu Y, Li X, Shan H. Evidence for gastrointestinal infection of SARS-Cov-2. Gastroenterology 2020;S0016-5085(20)30282-1; [Epub ahead of print.] 10.1053/j.gastro.2020.02.055 32142773

14 Wilson E, Caswell G, Turner N, Pollock K. Managing medicines for patients dying at home: a review of family caregivers' experiences. J Pain Symptom Manage 2018;56:962-74. 10.1016/j.jpainsymman.2018.08.019 30217417

15 Perkins E, Gambles M, Houten R, etal. The care of dying people in nursing homes and intensive care units: a qualitative mixed-methods study. Health Services and Delivery Research 2016;4. 10.3310/hsdr04200 27386612

16 Rosenberg JP, Bullen T, Maher K. Supporting family caregivers with palliative symptom management: a qualitative analysis of the provision of an emergency medication kit in the home setting. Am J Hosp Palliat Care 2015;32:484-9. 10.1177/1049909114531326 24803586

Published by the BMJ Publishing Group Limited. For permission to use (where not already granted under a licence) please go to http://group.bmj.com/group/rights-licensing/ permissions 